\title{
Lesões traumáticas de pele causadas pelos espinhos de Mimosa pudica e Mimosa debilis em equídeos ${ }^{1}$
}

\author{
Alessandra S. Belo Reis ${ }^{2}$, Marcos Dutra Duarte ${ }^{2}$, Melina G.S. de Sousa ${ }^{2}$, Nayra \\ Fernanda de Q.R. Freitas ${ }^{2}$, Elise M. Yamasaki ${ }^{3}$, André G. Marciel e Silva ${ }^{2}$, \\ Carlos Magno C. Oliveira² e José Diomedes Barbosa²
}

\begin{abstract}
Belo Reis A.S., Duarte M.D., Sousa M.G.S., Freitas N.F.Q.R., Yamasaki E.M., Silva A.G.M., Oliveira C.M.C. \& Barbosa J.D. 2011. [Traumatic skin injuries caused by the thorns of Mimosa pudica and Mimosa debilis in Equidae.] Lesões traumáticas de pele causadas pelos espinhos de Mimosa pudica e Mimosa debilis em equídeos. Pesquisa Veterinária Brasileira 31(9):768-772. Central de Diagnóstico Veterinário, Faculdade de Medicina Veterinária, Universidade Federal do Pará, Campus Castanhal, Rua Maximino Porpino da Silva 1000, Castanhal, PA 68740-080, Brazil. E-mail: alessandra.belo.reis@gmail.com

Studies on the nature and cause of skin lesions in horses on a farm in the county of Castanhal, northeastern Para, Brazil were conducted. These were visits to the farm, epidemiological studies, blood sample collections, skin biopsies, and inspection of the pasture. The study included 25 Equidae, 14 males and 11 females, six months to eight years old. The animals showed ulcerative lesions of irregular borders on the head (nose, muzzle, upper and lower lips), in the oral cavity (buccal vestibule and gum) and on the limbs (billets, metacarpals and metatarsals and scapular-humeral joint). The histopathological examination revealed foci of cutaneous erosions with epidermal necrosis, spongiosis and vesicular degeneration of the remaining epidermis, and mild inflammatory infiltrate in the underlying dermis, consisting predominantly of macrophages and, to a lesser degree, eosinophils. The inspection of the pasture, which consisted of Brachiaria humidicola, revealed a heavy invasion by two plants provided with spines, Mimosa pudica and Mimosa debilis, of the Leguminosae Mimosoideae family. It was concluded that the skin lesions were caused by traumatic action of the spines of Mimosa pudica and Mimosa debilis.
\end{abstract}

INDEX TERMS: Traumatizing plants, skin lesions, Mimosa pudica, Mimosa debilis, Equidae.

RESUMO.- Foram realizadas pesquisas sobre a natureza e causa de lesões de pele em equídeos em uma propriedade no município de Castanhal, região Nordeste do Estado do Pará. Foram realizadas visitas técnicas, estudos epidemiológicos, coletas de sangue, biópsias de pele afetada e a inspeção da pastagem. 0 estudo incluiu 25 equídeos, dos quais 14 machos e 11 fêmeas, de seis meses e oito anos de idade. Os animais apresentaram lesões ulcerativas, de bordos irregulares, na cabeça (narinas, focinho, lábios superiores e inferiores e chanfro), na cavidade oral (vestíbulo bucal e gengiva) e nos membros (boletos,

\footnotetext{
${ }^{1}$ Recebido em 22 de março de 2011.

Aceito para publicação em 29 de junho de 2011.

${ }^{2}$ Central de Diagnóstico Veterinário, Faculdade de Medicina Veterinária, Universidade Federal do Pará (UFPA), Campus Castanhal, Rua Maximino Porpino da Silva 1000, Centro, Castanhal, PA 68740-080, Brasil. *Autor para correspondência: alessandra.belo.reis@gmail.com

${ }^{3}$ Curso de Pós-Graduação em Ciência Veterinária, Universidade Federal Rural do Rio de Janeiro (UFRRJ), Seropédica, RJ 23890-000, Brasil.
}

metacarpos e metatarsos e articulação escápulo-umeral). No exame histopatológico foram observados focos de erosões cutâneas, caracterizados por perda e necrose da epiderme, com espongiose, degeneração vesicular da epiderme remanescente e leve infiltrado inflamatório na derme subjacente, constituído predominantemente por macrófagos e, em menor grau, eosinófilos. Na inspeção da pastagem, constituída de Brachiaria humidicola, foi constatada grande invasão de duas plantas providas de espinhos, Mimosa pudica e Mimosa debilis, ambas da família Leg. Mimosoideae. Concluiu-se, que as lesões de pele foram causadas pela ação traumática dos espinhos de Mimosa pudica e Mimosa debilis.

TERMOS DE INDEXAÇÃO: Plantas traumatizantes, lesões de pele, Mimosa pudica, Mimosa debilis, equídeos.

\section{INTRODUÇÃo}

As enfermidades cutâneas são frequentemente diagnosticadas e causam prejuízos para a criação dos equinos, além de 
ocasionar grandes perdas econômicas devido aos custos com tratamentos por vezes prolongados, resultam em uma aparência externa desagradável, dificultando inclusive o transporte e a utilização dos animais em provas hípicas e exposições (Radostits et al. 2002).

Existem vários fatores endógenos e exógenos que podem causar, potencialmente, agressão à pele. Os fatores endógenos envolvidos na agressão à pele são imunológico, congênito, hereditário, hormonal, metabólico e idade do animal. Já os fatores exógenos são nutricional, microbiano, químico, físico, parasitário e alérgico (Hargis \& Ginn 2009).

As feridas são classificadas quanto ao grau de penetração na pele em superficiais ou profundas. Feridas superficiais são as que não atingem a espessura total da pele e incluem equimoses, contusões e hematomas. Profundas, são feridas que penetram a derme e estão incluídas as incisões, lacerações e perfurações. As lesões mais comuns em equinos são as perfurações e lacerações, geralmente causadas por arames, pedaços de madeira ou mordidas (Thomassian 2005).

Este trabalho tem por objetivo estudar a natureza e a causa de lesões de pele em uma propriedade no município de Castanhal, região nordeste do Estado do Pará.

\section{MATERIAL E MÉTODOS}

Os dados epidemiológicos e clínico-patológicos foram obtidos através de visitas a uma propriedade no município de Castanhal, na região nordeste do Estado do Pará. Foi realizado exame clínico de 25 equídeos (23 equinos e dois muares), sendo 14 fêmeas e 11 machos, com seis meses a oito anos de idade. Foram realizadas coletas de sangue através de venopunção com agulhas 30x8 em tubos com vácuo, de $5 \mathrm{ml}$, com e sem EDTA. Os valores hematológicos foram determinados através de contador automático de células (CELM-CC-550).

O soro foi obtido através de centrifugação das amostras a $3.000 \mathrm{rpm}$ durante 5 minutos e em seguida foi acondicionado em tubos de polietileno (ependorf) de $1,5 \mathrm{ml}$ e congelados a $-20^{\circ} \mathrm{C}$ até a realização das análises. Foram realizadas análises de uréia, creatinina, aspartato aminotransferase (AST), alanina aminotransferase (ALT), gamaglutamiltransferase (GGT), bilirrubina direta e total, utilizando kits específicos (Cepa®), com leitura em espectofotômetro, para se descartar a possibilidade de fotossensibilização.

0 hemograma e a bioquímica sérica foram realizados no laboratório de Patologia Clínica da Central de Diagnóstico Veterinário (CEDIVET) da Universidade Federal do Pará.

Foram realizadas biópsias segundo Feitosa (2004) da pele afetada, da região da face, de quatro animais, que apresentaram lesões em variados estágios de evolução. 0 material foi processado pelo Setor de Patologia Animal, do Projeto Sanidade Animal da Universidade Federal Rural do Rio de Janeiro.

Foram coletadas amostras das plantas para identificação botânica, que foi realizada pelo Instituto de Biologia da Universidade Federal Rural do Rio de Janeiro.

\section{RESULTADOS}

Os equinos acometidos pertenciam às raças Árabe, Quarto de Milha e seus mestiços. Os muares eram filhos de égua Mangalarga com jumento Pega. Os animais eram criados de forma extensiva, em pastagem constituída de Brachiaria humidicola, e recebiam sal mineral específico para a espécie equina.

Dos 25 animais, 24 apresentaram lesões traumáticas na pele; destes, sete apresentaram lesões nos membros, na cabeça e cavidade oral, 11 na cabeça e nos membros, quatro

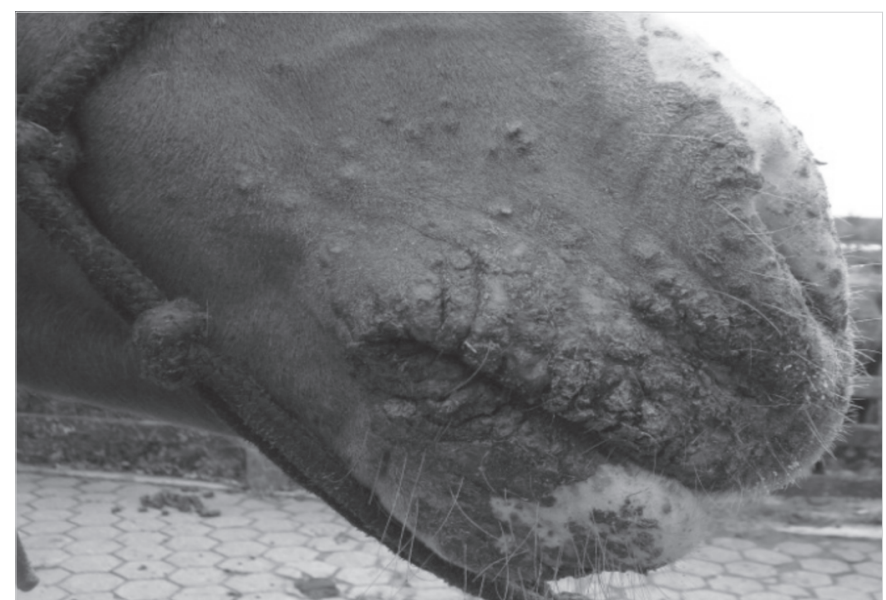

Fig.1. Equino 2 com lesões ulcerativas com bordos irregulares na pele da narina e dos lábios, causadas por Mimosa pudica e Mimosa debilis.

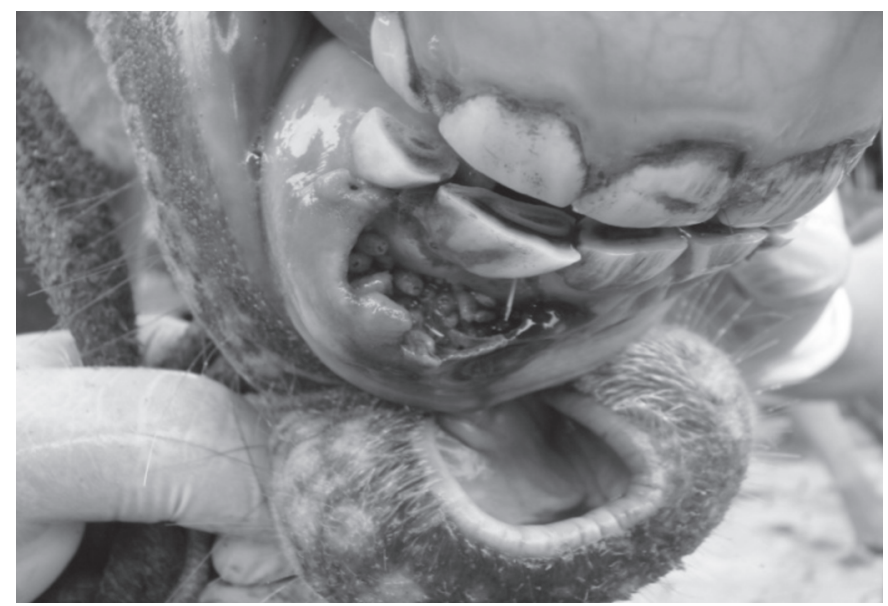

Fig.2. Lesões ulcerativas na gengiva do Equino 1, com presença de miíase na base dos incisivos inferiores, causadas por Mimosa pudica e Mimosa debilis.

somente na região da cabeça e dois somente na região dos membros.

As lesões na cabeça envolviam os lábios superiores e inferiores, focinho, narinas, bochechas e chanfro, e em alguns casos, envolviam a cavidade oral com lesões na gengiva e no vestíbulo bucal. As lesões na região da cabeça inicialmente se caracterizavam por pequenas ulcerações na pele que evoluíam para feridas maiores de contornos irregulares (Fig. 1). Na cavidade oral, no estágio inicial, observaram-se apenas pequenas escoriações e, nos casos mais avançados, havia perda de tecido na base dos dentes com formação de extensas feridas e a presença de miĺase (Fig.2).

Nos membros, as lesões estavam localizadas nos boletos; em alguns casos, se estendiam até a região dos metacarpos, metatarsos e a articulação escápulo-umeral. Na fase inicial das lesões foram observadas perda de pelos, pequenas escoriações com espinhos inseridos na pele e reação inflamatório ao redor (Fig.3). Nos casos moderados as lesões atingiam as camadas da epiderme e da derme, e nos casos graves, havia ainda o comprometimento do tecido subcutâneo e exsudação serossanguinolenta (Fig. 4 e 5). Em alguns casos foi observada também a presença de miíase. 

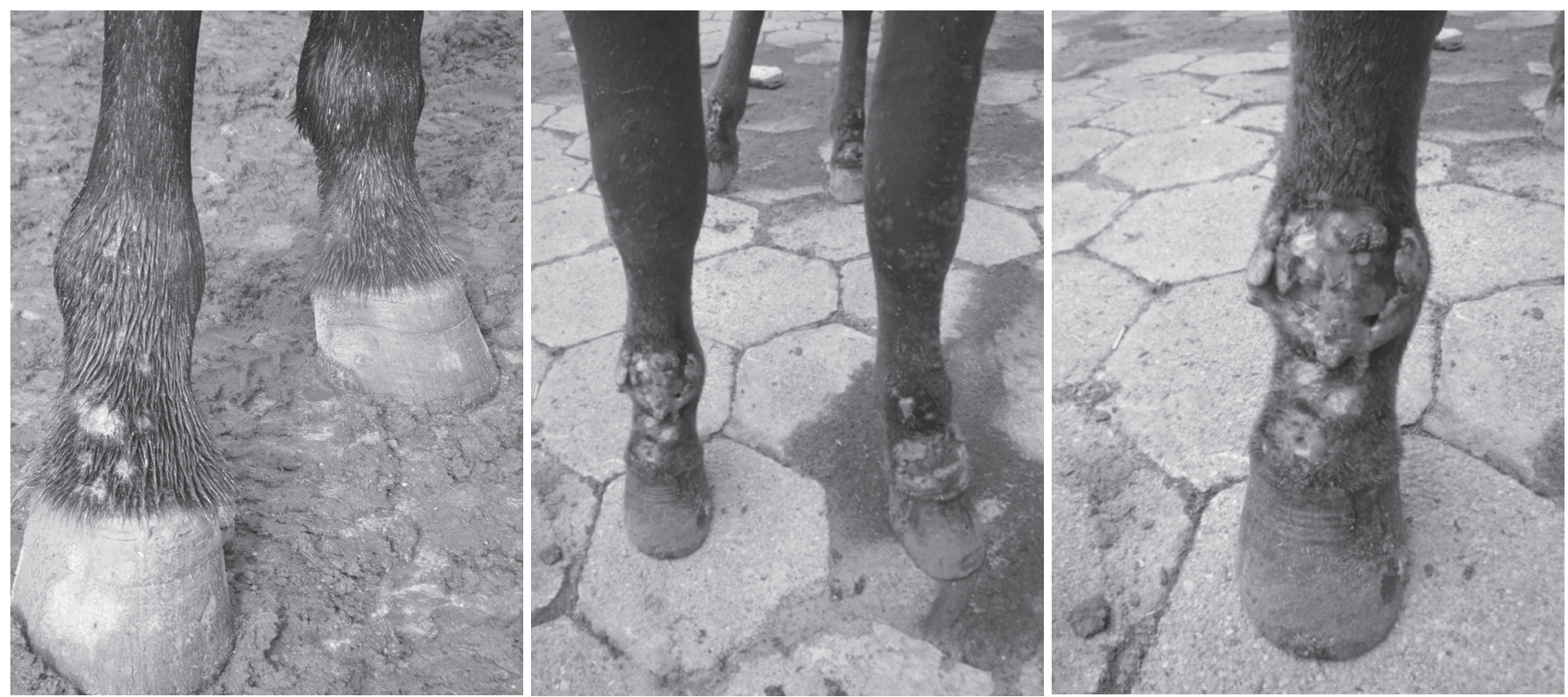

Fig.3. Lesões, em fase inicial, com perda de pelos e pequenas escoriações na pele da quartela e boleto do Equino 4, causadas por Mimosa pudica e Mimosa debilis.

Fig.4. Lesões ulcerativas nos quatro membros do Equino 1, atingindo a pele e tecido subcutâneo, com exsudação serossanguinolenta e edema nos membros torácicos, causadas por Mimosa pudica e Mimosa debilis.

Fig.5. Detalhe da figura anterior: Membro anterior direito, com lesões na região da quartela e boleto.

À inspeção da pastagem constatou-se grande quantidade de duas plantas traumatizantes com caules providos de grande quantidade de espinhos, que foram identificadas como $\mathrm{Mi}$ mosa pudica e Mimosa debilis, ambas da família Leguminosae Mimosoideae (Fig.6-9).

Foi sugerido ao proprietário, de transferir os animais para um piquete sem as duas plantas traumatizantes. No decorrer de três semanas, após a formação de tecido de granulação, havia a cicatrização das lesões. Após a cicatrização, ocorreu despigmentação da pele nas áreas correspondentes às feridas na região da cabeça (Fig.10).

Dos 24 animais que apresentaram lesões de pele, seis, com lesões graves, apresentaram anemia. As concentrações das enzimas hepáticas e renais apresentaram-se dentro dos parâmetros normais para a espécie equina.

No exame histopatológico dos fragmentos obtidos por biopsia foram observados focos de erosões cutâneas, caracterizados por perda e necrose da epiderme, com espongiose e degeneração vesicular da epiderme remanescente e leve infiltrado inflamatório na derme subjacente, constituído predominantemente por macrófagos e, em menor grau, por eosinófilos (Fig.11).

\section{DISCUSSÃO E CONCLUSÕES}

A flora brasileira é uma das mais diversificadas do planeta. Plantas traumatizantes, alergênicas e venenosas são encontradas em todo o país acometendo humanos e animais (Haddad Junior 2004). Os mecanismos nocivos mais comuns relatados em humanos são traumáticos e reações alérgicas (Scott \& Thomas 1997). Nos animais, os mecanismos conhecidos ocorrem pela ingestão de plantas que contêm substâncias tóxicas (Tokarnia et al. 2000) e pela ação traumática (Vaz et al. 1998, Barbosa et al. 2009a,b,c). Na África do Sul já foram descritas várias plan- tas que possuem espinhos ou frutos, os quais causam traumatismo na pele e em outras partes do corpo de bovinos e ovinos, com danos consideráveis à saúde, inclusive morte (Kellerman et al. 2005). No Brasil, existem poucos trabalhos referentes a plantas que causam lesões traumáticas em animais de produção (Vaz et al. 1998, Barbosa et al. 2009a,b,c). Em equinos foram relatadas lesões na comissura labial causadas pela ação traumática das folhas de Panicum maximum, denominada de queilite angular traumática (Barbosa et al. 2009b).

Nos últimos anos plantas invasoras conhecidas popularmente como "dorme-maria", "dormideira", "arranhadeira", "malícia" e "não-me-toque", vêm se difundindo nas pastagens localizadas na região nordeste do estado do Pará. Trata-se de arbustos perenes comuns na América tropical, e que se adaptam bem em locais úmidos, terrenos baldios, gramados e plantações abertas (Lorenzi \& Matos 2008).

São plantas que se difundem rapidamente e os produtores rurais que não têm recursos ou conhecimento sobre o controle dessas plantas, não conseguem impedir a sua proliferação e acabam abandonando as pastagens invadidas. Recentemente, uma dessas plantas, Mimosa pudica, que tem espinhos, foi identificada como causadora de problemas de pele nos membros de ovinos e bovinos que pastavam em piquetes severamente invadidos (Barbosa et al. 2009a). As lesões de pele nestes animais foram semelhantes às estudadas no presente trabalho, porém, envolviam somente os membros. No presente estudo, além dos membros, foram afetadas a pele da cabeça e a mucosa da cavidade oral o que ocorreu provavelmente devido aos arbustos de Mimosa debilis serem mais altos que os de Mimosa pudica, e estes, por encobrirem a pastagem, obrigavam os animais a colocarem a cabeça por entre estes arbustos para se alimentarem, traumatizando, dessa forma, a pele da região da cabeça e a cavidade oral. 

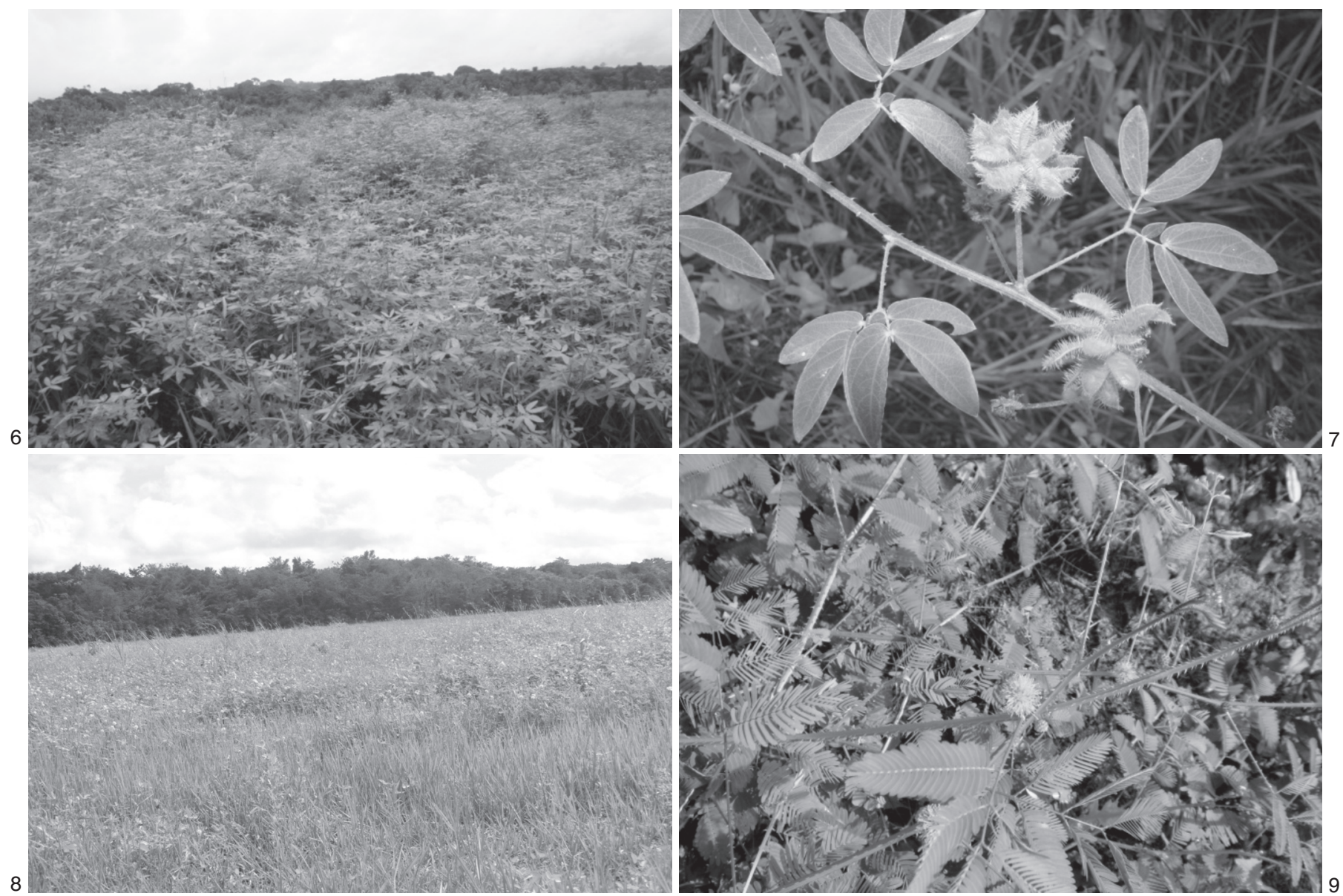

Fig.6. Parte da pastagem severamente invadida por Mimosa debilis. Castanhal, PA.

Fig.8. Outra parte da pastagem severamente invadida por Mimosa pudica. Castanhal, PA.

Na fase de cicatrização das lesões nas regiões dos membros, nos equinos, foi observada formação de tecido de granulação, que segundo Ackermann (2009) é uma reação comum na pele de equinos, sendo formado por tecido conjuntivo, fibroblastos e vasos sanguíneos.

Foi observada também despigmentação de pele na região



Fig.10. Lesões em fase de cicatrização com presença de crostas e com áreas despigmentadas nas regiões da narina e dos lábios do Equino 6, causadas por Mimosa pudica e Mimosa debilis.



Fig.7. Aspecto das folhas, caules com espinhos e vagens de Mimosa debilis.

Fig.9. Aspecto das folhas, caule com espinhos e inflorescências de Mimosa pudica.

da face, alteração que, segundo Scott \& Miller (2004), é comum em cavalos e ocorre geralmente após a regressão de papilomas, inflamações, complicação da oncocercose, lúpus eritematoso ou queimaduras. Segundo White \& Evans (2006) a despigmentação da pele é comumente associada a traumatismo e inflamação, particularmente em equinos.



Fig.11. Destruição do epitélio acompanhado de infiltrado inflamatório na derme subjacente, constituído predominantemente por macrófagos e, em menor grau, por eosinófilos. 
Nos animais com lesões graves, foi observada anemia, que pode ser justificada pelo fato das feridas serem extensas e delas fluir líquido serossanguinolento, com perda de hemácias (Thrall 2007).

Deve-se realizar o diagnóstico diferencial com outras doenças que causam lesões de pele em equídeos, como fotossensibilização hepatógena e pitiose.

Na fotossensibilização hepatógena, os animais apresentam lesões com desprendimento da pele, preferencialmente em regiões despigmentadas (Schild 2007). Além dessas lesões de pele, os animais também apresentam lesão hepática, que pode ser detectada através do aumento das enzimas hepáticas (Barbosa et al. 2006). No presente trabalho, estes valores se apresentaram dentro dos parâmetros normais para a espécie equina.

Na pitiose, em lesões recentes, observam-se pequenas áreas elevadas desprovidas de pelos, com ulceração da pele e exsudação de líquido serossanguinolento, porém, as lesões evoluem rapidamente, têm crescimento progressivo e podemse observar os "kunkers" (massa necrótica de coloração amarelada e endurecida) (Pereira \& Meireles 2007).

Baseado no conjunto dos dados, ou seja, na presença de grande quantidade dessas plantas traumatizantes na pastagem, na recuperação dos animais depois da retirada da área invadida, nas lesões ulcerativas na pele e cavidade oral e nos achados histopatológicos, concluímos que as lesões descritas neste estudo, foram causadas pela ação traumática de Mimosa pudica e Mimosa debilis.

Agradecimentos.- Ao Prof. Pedro Germano Filho, Instituto de Biologia da Universidade Federal Rural do Rio de Janeiro (UFRRJ), pela identificação do material botânico e ao proprietário da Fazenda Itaqui.

\section{REFERÊNCIAS}

Ackermann M.R. 2009. Inflamação crônica e cicatrização de feridas, p.183. In: McGavin M.D. \& Zachary J.F. (Eds), Bases da Patologia em Veterinária. $4^{\mathrm{a}}$ ed. Elsevier, Rio de Janeiro. 1476p.

Barbosa J.D., Oliveira C.M.C., Tokarnia C.H. \& Peixoto P.V. 2006. Fotossensibilização hepatógena em eqüinos pela ingestão de Brachiaria humidicola no estado do Pará. Pesq. Vet. Bras. 26:147-153.

Barbosa J.D., Silveira J.A.S., Albernaz T.T., Silva N.S., Belo Reis A.S., Oliveira C.M.C., Riet-Correa G. \& Duarte M.D. 2009a. Lesões de pele causadas pelos espinhos de Mimosa pudica (Leg. Mimosoideae) nos membros de bovinos e ovinos no estado do Pará. Pesq. Vet. Bras. 29(5):435438.

Barbosa J.D., Albernaz T.T., Riet-Correa G., Cerqueira V.D., Soares S.O., Campos K.F., Oliveira C.M.C. \& Duarte M.D. 2009b. Queilite angular traumáti- ca em eqüinos associada à ingestão de Panicum maximum. Pesq. Vet. Bras. 29(5):428-430.

Barbosa J.D., Junior J.M.A., Pinheiro C.P., Oliveira C.H.S., Oliveira C.M.C., Sousa M.G.S. \& Duarte M.D. 2009c. Acúmulo de sementes da palmeira Acrocomia aculeata nas bochechas de bubalinos, no estado do Pará. Pesq. Vet. Bras. 29(5):435-438.

Feitosa F.L. 2004. Semiologia Veterinária: a arte do exame clínico. Roca, São Paulo. 807p.

Ferreira G.C. \& Andrade A.C.S. 2006. Diretrizes para coleta, herborização e identificação de material botânico nas parcelas permanentes em florestas naturais da Amazônia brasileira. Disponível em <http://www.cnpf. embrapa.br> Acesso em 22 mar. 2010.

Haddad Junior V. 2004. Skin manifestations caused by Brazilian traumatic, allergenic, and venomous plants: Main species, therapeutic and preventive measures. J. Venom. Anim. Toxins incl. Trop. Dis. 10(3):206.

Hargis A.N. \& Ginn P.E. 2009. As respostas da pele à lesão, p.1125. In: McGavin M.D. \& Zachary J.F. (Eds), Bases da Patologia em Veterinária. $4^{\underline{a}}$ ed. Elsevier, Rio de Janeiro. 1476p.

Kellerman T.S., Coetzer J.A.W., Naudé T.W. \& Botha C.J. 2005. Plant Poisonings and Mycotoxicoses of Livestock in Southern Africa. $2^{\text {nd }}$ ed. Oxford University Press, Cape Town. 310p.

Lorenzi H. \& Matos F.J.A. 2008. Plantas Medicinais no Brasil: nativas e exóticas. $2^{\mathrm{a}}$ ed. Instituto Plantarum de Estudos da Flora, Nova Odessa. 544p.

Pereira D.B. \& Meireles M.A. 2007. Pitiose, p.457-466. In: Riet-Correa F., Schild A.L., Lemos R.A.A. \& Borges J.R.J. (Eds), Doenças de Ruminantes e Eqüídeos. Vol.1. $3^{\mathrm{a}}$ ed. Pallotti, Santa Maria, RS. 719p.

Radostits O.M., Gay C.C., Blood D.C. \& Hinchcliff K.W. 2002. Clínica Veterinária. Um tratado de doenças dos bovinos, ovinos, suínos, caprinos e equinos. 9a ed. Guanabara koogan, Rio de Janeiro. 1737p.

Schild A.L. 2007. Fotossensibilização hepatógena, p.39-42. In: Riet-Correa F., Schild A.L., Lemos R.A.A. \& Borges J.R.J. (Eds), Doenças de Ruminantes e Eqüídeos. Vol.2. 3aa ed. Pallotti, Santa Maria, RS. 694p.

Scott D.W. \& Miller W.H. 2004. Dermatologia Equina. Inter-Médica, Buenos Aires. 719p.

Scott S. \& Thomas C. 1997. Poisonous Plants of Paradise: First aid and medical treatment from Hawaii's plants. University of Hawaii's Press, Honolulu. 178p.

Thomassian A. 2005. Enfermidades dos Cavalos. 4ª ed. Varela, São Paulo. 573p.

Thrall M.A. 2007. Hematologia e Bioquímica Clínica Veterinária. Roca, São Paulo. 582p.

Tokarnia C.H., Döbereiner J. \& Peixoto P.V. 2000. Plantas Tóxicas do Brasil. Editora Helianthus, Rio de Janeiro. 310p.

Vaz C.M.S.L., Muniz E.N., Borba M.F.S. \& Oliveira O.L.P. 1998. Incidência de lesões na pele de cordeiros Corriedale e Ideal causadas por flechilha (Stipa $\mathrm{sp})$. Anais 35a Reunião Anual da Sociedade Brasileira de Zootecnia, Botucatu, SP, vol.3. p.34-36.

White S.D. \& Evans A.G. 2006. Pigmentação anormal, p.204-206. In: Smith B.P. (Ed), Medicina Interna de Grandes Animais. $3^{\underline{a}}$ ed. Manole, Barueri, SP. 1728 p. 